\title{
ENTRETEJIENDO COMUNIDADES Y UNIVERSIDADES: DESAFÍOS EPISTEMOLÓGICOS ACTUALES
}

\section{INTERWEAVING COMMUNITIES AND UNIVERSITIES: CURRENT EPISTEMOLOGICAL CHALLENGES}

\section{Resumen}

Zayda Sierra

El propósito de este artículo es problematizar conceptualmente e invitar a la discusión sobre la tendencia dominante de la universidad en el contexto global, y particularmente en América Latina, en reproducir un status quo que hace invisibles perspectivas e ideas alternativas sobre nociones de desarrollo comunitario y sostenibilidad desde nuevos imaginarios; los cuales cuestionan los parámetros culturales, políticos y económicos definidos por la noción eurocéntrica de modernidad y que contribuyen a replantear el quehacer investigativo. Presentaremos aquí desafíos conceptuales específicos que venimos enfrentando en el proceso de creación de un proyecto internacional entre una universidad colombiana y otra canadiense, con organizaciones comunitarias de base étnicas y rurales colombianas y con apoyo de organizaciones no gubernamentales, para abordar asuntos de sostenibilidad y educación desde una perspectiva decolonial.

Palabras clave: Paradigmas socioculturales, políticas de educación superior, participación comunitaria, poblaciones étnicas y rurales, perspectivas decoloniales

\section{Abstract}

The purpose of this conceptual paper is to problematize and engage the readers in a discussion about the dominant trend in higher education around the world, and particularly in Latin America, in reproducing the status quo; making invisible alternative perspectives and insights that could reshape and 
empower research on notions of community development and sustainability with new imaginings that question the cultural-political-economic space defined by Euro centered notion of modernity. We will share the specific conceptual challenges we are facing while developing an international research project between rural community-based organizations, NGOs, and Colombian and Canadian universities, addressing issues of sustainability and education within Colombian Afro-descendent, Campesino and Indigenous contexts from a decolonial perspective.

Key Words: Sociocultural Paradigms, Higher Education Policy, Community Engagement, Ethnic and Rural Populations, Decolonial Perspectives

\section{INTRODUCCIÓN}

La autora y el autor de este artículo, aunque procedemos de diferente latitud geográfica y socio-cultural, coincidimos en nuestra preocupación por el impacto de las políticas educativas neoliberales actuales en la misión social y los objetivos de las instituciones post-secundarias, en especial con aquellas voces que han sido marginadas de la academia: poblaciones indígenas, afro y campesina, para el caso colombiano. A lo largo y ancho del mundo de la política pública para la educación superior, sistemáticamente observamos cómo perspectivas académicas dominantes limitan o niegan el espacio para la construcción de conocimiento de comunidades históricamente excluidas. Nos interesa contribuir para que estas otras perspectivas sean consideradas en la educación superior, así como problematizar las relaciones de poder en la generación de conocimiento de personas del mundo académico que, como nosotros, no venimos de estos contextos marginados. ¿Cómo lograr se abran diálogos y discusiones más equitativas que permitan reconocer a esa Otra y ese Otro diferente quien a su vez tiene sus propios planteamientos y propuestas sobre educación superior? Nuestra preocupación no es tanto cómo lograr que otros sistemas de conocimiento permeen la educación superior sino cómo formas hegemónicas de conceptualizar y hacer investigación constriñen los prospectos para la acción de aquellos que están por fuera de los campos epistémicos y ontológicos eurocéntricos.

En este artículo compartimos algunas reflexiones que contribuyan a replantear la investigación sobre objetivos y políticas en educación superior 
desde otros imaginarios, aquellos que cuestionan fundamentalmente el espacio cultural, político y económico definido por una noción eurocéntrica de modernidad y posmodernidad. Somos conscientes que abogar por la presencia de sistemas de conocimientos indígenas, afro y campesinos en los objetivos de la educación superior colombiana eleva serias inquietudes sobre nuestra legitimidad y autoridad por no ser originarios de estas poblaciones. Aunque nos es claro que solamente los pueblos indígenas, afro y campesinos pueden legítimamente determinar sus identidades colectivas $-y$ como éstas deban darle forma a programas educativos, servicios y concepciones de educación superior-, no podemos desconocer que nos corresponde una enorme responsabilidad como investigadores de la sociedad dominante para que la academia y la sociedad se interrogue sobre sus propios marcos de referencias y concepciones culturales, particularmente en relación a pueblos que han sido históricamente excluidos de espacios de comprensión de su propio trabajo, tanto teórico como práctico, en distintos campos del saber, así como de poder pensar y proponer la misión y propósitos de la educación superior.

Nuestra discusión sobre cómo pensar la educación superior se basa en lo que pensamos es la naturaleza sociocultural y por ende política de una sociedad. Comprender qué entendemos por paradigmas socioculturales es crucial si queremos ver cómo diferentes concepciones de educación superior se expresan y reflejan en las políticas educativas, sean éstas informales, latentes o implícitas a la vez que formales. Avanzar hacia una mayor comprensión de las bases paradigmáticas socioculturales y educativas respecto a distintas concepciones de educación superior es también esencial para poder establecer escenarios distintos que vayan más allá de las ideologías dominantes individualistas, consumistas y de mercantilización o comodificación actual de la educación superior (Ball, 2012).

En este artículo esperamos vincular a lectoras y lectores en un diálogo que desafíe la intolerancia actual hacia otras cosmovisiones u ontologías de carácter relacional, a la vez que contribuya con la coexistencia equitativa y la construcción conjunta de saberes. Al respecto abordaremos dos preguntas que consideramos centrales para repensar la educación superior desde perspectivas indígenas, afrodescendientes o campesinas: (1) ¿desde cuáles paradigmas socioculturales y marcos epistémicos (modos de conocer) debemos enfocar nuestras relaciones entre las personas y entre ellas y la naturaleza, que nos abra espacios de experiencias plurales que nos permitan imaginar otros mundos posibles más allá de la modernidad? (Escobar, 2004, p. 213); y (2) ¿cómo 
estos otros paradigmas pueden apoyarnos en la formulación de propuestas de educación superior que contribuyan con los esfuerzos de poblaciones históricamente excluidas hacia la constitución de mundos alternativos, distintos a los modelos eurócentricos y coloniales dominantes?

\section{PARADIGMAS DIVERSOS EN EDUCACIÓN SUPERIOR Y SU IMPACTO EN COMUNIDADES ÉTNICAS Y RURALES}

Las universidades -como espacios de concentración, reproducción y creación de saber - cumplen una función central en la sociedad moderna, tanto en el entrenamiento de talento humano especializado como en la formación de personas o grupos de personas con la capacidad de incidir en políticas locales, nacionales o globales. Para nuestro análisis planteamos que la educación superior, como cualquier otro proceso educativo, es expresión de distintos paradigmas socioculturales desde los cuales se orientan sus objetivos. Bertrand y Valois (1980, citados por Paquette y Fallon, 2010) definen un paradigma sociocultural como,

La acción ejercida por una sociedad, y como resultado de su actividad, en sus prácticas sociales y culturales, mediante la combinatoria de cinco elementos: 1) concepción de conocimiento; 2) concepción de las relaciones entre las personas, la sociedad y la naturaleza; 3) un sistema de valores, 4) una manera de hacer las cosas, manejo y toma de decisiones; $y, 5$ ) un sentido predominante de enfocar la existencia. Cada paradigma sociocultural enmarca una concepción específica de educación (paradigma educativo), que define las normas y reglas que determinan la reflexión y acción educativa, por un lado, y la praxis que promueve modalidades particulares con relación a metodologías de enseñanza y otras. (p. 24-25).

Para Bertrand y Valois, entre los paradigmas dominantes actuales estarían el 1) paradigma industrial $\boldsymbol{y}$ postindustrial, en el cual el individuo vale si está integrado al proyecto económico de la sociedad moderna basado en el mercado, el consumo y la competitividad, y en el cual la ganancia individual prevalece sobre el bien común; y el 2) paradigma existencial o humanista, que propone facilitar el desarrollo pleno del potencial creativo de cada individuo para que sea funcional en una sociedad que se presume puede ser mejorada con la activa participación de cada persona, cuya libertad de expresión es la que permite enfrentar cualquier estructura autoritaria, aunque ello no significa necesariamente el mejoramiento o la transformación de la sociedad sino 
defender el status quo.

Podemos relacionar los paradigmas anteriores con el 3) paradigma neolibera/mediante el cual se considera que son los individuos emprendedores el motor del progreso y no los trabajadores o la asociación y cooperación social. La injerencia del Estado debe reducirse a su mínima expresión pues coarta la capacidad individual emprendedora; se acepta la intervención del Estado sólo en casos de catástrofes naturales, y se espera que sea el sector privado quien se encargue del manejo de los subsidios y hacer de los pobres agentes de mercado. El neoliberalismo desconfía del "bien común", del "interés colectivo" y de la "justicia social." Al ignorar la historia y predicar como natural la libertad individual, el neoliberalismo termina justificando la opresión, el sometimiento y la pérdida de toda posibilidad de libertad para la mayoría de individuos (Restrepo, 2003: 21-22).

Desde estos paradigmas industrial, existencial y neoliberal, retomando nuevamente a Bertrand \& Valois (1980), el principal objetivo de la educación superior sería iniciar al individuo en la idea de desarrollo y consumo, promoviendo una visión de la creatividad como sinónimo de progreso económico, científico y tecnológico. La educación superior ve entonces las relaciones humanas en términos de relaciones económicas y promueve la imagen de una persona deseable como alguien que es "oportunista, materialista y conformista" (no lejos de planteamientos contemporáneos sobre "educación empresarial", véase, por ejemplo, Smyth, 1999). Políticamente, la educación dentro de paradigmas industriales y neoliberales debe contribuir a mantener una estructura oligárquica social, la aceptación de que una minoría [élite] debe tomar decisiones en nombre de la mayoría. En definitiva, la educación debe reflejar y legitimar una estructura jerárquica de toma de decisiones. En términos del orden socio-económico, la educación debe "relativizar la importancia del estudiante como persona, mientras maximiza su importancia como futuro trabajador." Así mismo, la educación debe "promover aptitudes intelectuales que contribuyan a la reproducción de las divisiones sociales [existentes] en el trabajo y promover la legitimidad del orden establecido y sus valores" (Bertrand \& Valois, 1980:173). No es sorprendente entonces que el objetivo general de la educación superior así concebida sea, por tanto, una contribución al mantenimiento del status quo de la industria, la sociedad postindustrial, o neoliberal, y la sociedad moderna en su conjunto.

Los paradigmas socioculturales industrial, humanista y neoliberal, si bien retratan la sociedad imperante hoy día, son insuficientes para interpretar de 
manera adecuada contextos dependientes de las metrópolis anglo-europeas, como es el caso de Latinoamérica. Retomaremos acá los aportes del colectivo de investigación en colonialidad/modernidad (Lander, 2000; Escobar, 1998; 2007; Walsh, 2009) y las discusiones de Castro-Gómez (2005) sobre el concepto de Imperio de Hardt y Negri (2001) que nos permiten reconocer al 4) paradigma colonial e imperial, desde el cual, bajo el discurso de la modernización, el progreso y el desarrollo, se lideran economías extractivas y de monocultivos en naciones denominadas subdesarrolladas o del "Tercer Mundo" y se justifica la apropiación de territorios enteros de pueblos originarios mediante jerarquías raciales, desplazando y proletarizando a comunidades agrarias, poniendo en riesgo la seguridad alimentaria de amplias capas de la población y la diversidad biocultural y social.

"El surgimiento de Imperio como principio de ordenación que cada vez más rige la producción, el procesamiento, la distribución y el consumo de alimentos, es el que contribuye al avance de lo que parece una crisis agraria inevitable. Esto se debe a que Imperio actúa como una explotación ecológica y socioeconómica desequilibrada. Imperio implica una degradación de la naturaleza, de los agricultores, de los alimentos y de la cultura. El capital ecológico, social y cultural se va desmoronando" (Ploeg, 2010:34)

Para Castro-Gómez (2005), Imperio no conlleva el fin de la colonialidad sino su reorganización posmoderna; esta reorganización imperial de la colonialidad es la otra cara que el Imperio necesita para su consolidación. Reconocer el paradigma colonial e imperial nos obliga a descentrar la historia que nos han contado y reconocer que el fortalecimiento del mundo anglo-europeo no habría sido posible sin el aporte de la "periferia", tanto como fuente principal de riquezas naturales como de trabajo barato; así develar la relación constitutiva entre capitalismo y colonialismo (Coronil 2000).

En resistencia y oposición a estos paradigmas dominantes (o "paradogmas", como los llama Walsh, 2010; también paradigmas androcéntricos y patriarcales, ver Harding 1996; Lamus, 2012), han emergido otras posibilidades que día a día toman mayor relevancia como (5) paradigmas decoloniales, que nos invitan a un análisis crítico del funcionamiento del proyecto moderno y su concreción actual -la globalización y sus efectos- para buscar otras opciones a sus designios, mediante la construcción de redes locales/globales desde una alteridad políticamente responsable. Los proyectos decoloniales se construyen pory conlos grupos subalternizados; es acá donde este paradigma se constituye 
en una ética de la liberación (Palermo, 2010: 52-53). Es de resaltar aquí el 6) paradigma del Buen Vivir(Sumak Kawsay) o el Vivir Bien(Suma Qamaña), aporte de filosofías Quichua de Ecuador y Aymará de Bolivia, recientemente incluido en reformas constitucionales de estos países, y que viene siendo retomado por otros movimientos indígenas y sociales en el mundo. Este paradigma está orientado hacia la construcción de sociedades más justas y equitativas, que reconozcan el derecho a una vida digna de todas las personas así como de la naturaleza (Choquehuanca, 2010; Caudillo, 2012; Gudynas, 2011). El Buen Vivir, en su sentido más amplio,

Organiza y construye un sistema de conocimiento y vida basado en la comunión entre humanos y naturaleza, en una totalidad espacial-temporal armoniosa de existencia; esto es, en la necesaria interrelación de seres, conocimientos, lógicas y racionalidades de pensamiento, acción, existencia y vida. Esta noción es parte y componente de la cosmovisión, cosmología, o filosofía de los pueblos indígenas de Abya Yala, pero también, y en una manera diferente, de los descendientes de la Diáspora Africana (Walsh, 2010: 18).

Podemos correlacionar los paradigmas decolonial y del Buen Vivir con el 7) paradigma simbiosinérgico (Bertrand y Valois, 1980; citados por Paquette y Fallon, 2010: 31-32), el cual reconoce la imbricada relación entre las personas, la sociedad y la naturaleza como un ecosistema integrado. Bertrand and Valois retoman aquí el pensamiento de pueblos aborígenes norteamericanos, que nos recuerdan una y otra vez que la persona no es un ser aislado en el universo; a su vez las críticas a la sociedad industrial monocultural contemporánea por sus efectos negativos en la vida de diversos pueblos y ecosistemas. Desde esta perspectiva, la educación superior debe contribuir con la creación de "nuevas comunidades democráticas pluralistas": cada persona hace parte de un todo, el desarrollo personal debe resituarse dentro del desarrollo de la comunidad y, a su vez, el desarrollo de la comunidad debe contribuir con el desarrollo personal. Ello significaría profundizar en tecnologías contestatarias o blandas que contribuyan con la producción propia de los elementos físicos necesarios para la existencia; o, en discursos ecologistas actuales, lo que se viene proponiendo como futuro sostenible en oposición a la hiper-tecnificación e hiper-mercantilización de la vida social (Escobar 2004, p. 209).

Diversos movimientos sociales y epistémicos contra-hegemónicos (indígenas, postcoloniales, antirracistas, feministas, ecologistas, ecofeministas, agroecológicos, en defensa de los derechos humanos, por el desarrollo endógeno, por el "decrecimiento", entre otros) vienen aportando a 
la construcción de estos paradigmas decoloniales y simbiosinérgicos, aunque todavía su impacto en cambios políticos, económicos y sociales vaya más lento de lo esperado. Clarificar contradicciones, crear potenciales alianzas y construir esfuerzos conjuntos desde los aspectos solidarios de estos distintos movimientos y paradigmas alternativos deberá ser parte de la formación universitaria, si queremos alcanzar transformaciones más justas y equitativas en la relación entre los seres humanos y con la naturaleza; esto es, si queremos hacer viables "otros mundos posibles".

\section{FORMACIÓN INDIVIDUALISTA VS COMUNITARIA: PERTINENCIA DE LOS PROGRAMAS UNIVERSITARIOS EN CONTEXTOS RURALES Y ÉTNICOS}

El estudio de estos distintos paradigmas socioculturales nos sirve como ejercicio para examinar las propuestas educativas que se transmiten actualmente en los distintos programas universitarios: ¿qué tan relevantes son para comunidades en contextos étnicos y rurales? Desde los paradigmas 1 a 4 vemos que se privilegia una formación individualista y competitiva, que basa el éxito económico y político de las personas en la ganancia personal o corporativa, sin importar las consecuencias en el deterioro de la vida de pueblos enteros y el agotamiento de los ecosistemas. Para Glaveanu (2009), los discursos predominantes sobre creatividad consideran a los sujetos que lideran como personalidades "únicas" (el paradigma-Él) y tomados por separado (el paradigma-Yo). En cambio, perspectivas más integrales y complejas sobre la creatividad y el liderazgo deben incorporar múltiples niveles, desde los individuos y sus interacciones interpersonales, hasta los diversos grupos, culturas y ecosistemas (el paradigma-Nosotros). De acuerdo con este autor,

\footnotetext{
"Para aquellos con pretensiones universalistas y de modelos únicos de creatividad, esta perspectiva [el paradigma-Nosotros] puede ser decepcionante. No tiene "fórmulas" y promueve el estudio contextual y situado de las acciones, personas y comunidades creativas... Esta nueva visión significa (...) un cambio en nuestro proyecto cultural de dominar el medio ambiente para generar relaciones más creativas y de cuidado dentro de él. Es un recordatorio de nuestra responsabilidad como miembros de la comunidad para construir espacios de diálogo y creatividad tanto para sí mismos como para los demás". (Glaveanu, 2009:13)
}

El modelo universitario imperante nos presenta teorías y prácticas científicas como esfuerzos de grandes individuos y como si fueran de carácter 
"universal" y "neutral" cuando realmente conllevan relaciones de poder, al establecer conceptos como los de "superioridad" y "subdesarrollo" que sirven para deslegitimar otras maneras de ser, pensar y habitar el mundo, subsecuentemente justificando la dominación de unas personas y naciones sobre otras. Prácticas de conocimientos nativos son sistemáticamente reprimidas (violencia epistémica), mientras se mantiene alejado de los claustros universitarios a quienes tengan ancestros negros e indígenas (CastroGómez, 2000; Nieto, 2000). La relación entre poder y conocimiento no puede explicarse entonces como dos cosas ajenas que pueden interactuar, sino que hay que reconocer al poder mismo como la esencia del conocimiento:

\footnotetext{
"La ciencia y la medicina se han considerado símbolos de una superioridad cultural de los países occidentales sobre el llamado «nuevo mundo». Esta idea supone que la ciencia y la medicina son fuerzas históricas necesariamente progresivas, que las colonias tienen la fortuna de haber recibido "la luz» de la ciencia, y que por ello las culturas americanas deben sentirse obligadas y agradecidas con Europa". (Nieto, 2000:241).
}

En la actualidad, la mayoría de capacitaciones y certificaciones profesionales que se ofrecen a estudiantes de contextos rurales en América Latina están aisladas de sus escenarios comunitarios, y priman perspectivas coloniales y desarrollistas que presentan a las poblaciones étnicas y campesinas como atrasadas y carentes de progreso. Por el carácter monocultural de los contenidos universitarios, los pocos estudiantes que provienen de comunidades étnicas y campesinas desertan al no encontrar referentes académicos relacionados con sus contextos de vida, mientras que los pocos que se gradúan no retornan a sus comunidades de origen pues no ven allí espacios de aplicación de lo aprendido (Pancho et al, 2005; PNUD, 2011; Sierra 2005; UNESCO/IESALC, 2003).

Aunque paulatinamente se han venido creando programas de Acción Afirmativa para facilitar el acceso a la educación superior de poblaciones históricamente marginadas, siguen imperando modelos asimilantes y aculturizantes. Esto es, quienes vienen de comunidades étnicas y rurales deben acomodarse al esquema universitario existente, así ingresen por procesos de admisión especial, pues ni los currículos ni las prácticas académicas han sufrido cambios sustanciales que les permitan participar activamente en los procesos organizativos de sus comunidades, profundizar en la complejidad de sus culturas y abordar las presiones globales que les afectan hoy. Existen también críticas a la co-optación de propuestas educativas propias y a la utilización de las comunidades étnicas y rurales como fuentes de conocimiento y objeto de 
investigación, no como activas constructoras de saberes (Colectivo Intercultural, 2007, García, Pulido y Montes, 1999; León, 2005; Rebolledo, 2005). Ilustramos lo anterior con la descripción del historiador afrodescendiente Sergio Mosquera, sobre una universidad en la región del Pacífico colombiano:

"La Universidad nace sin una identidad étnica, nace más bien como un clon de la universidad occidental... no se orienta desde su creación al conocimiento de la realidad donde está asentada, para proponer solución a sus problemas... fue una Universidad que no fue pensada para la región ni para su gente [de mayoría afrochocoana]. También, a veces en la región, vamos a encontrar ese problema: de una descontextualización total de la Universidad en sus carreras, en sus programas, en sus planes, en su extensión a la comunidad. [...] Entonces habíamos desconocido que estábamos en un contexto donde compartíamos un territorio con otros grupos étnicos, y justamente se reproducía la conceptualización que tenemos de los grupos minorizados a nivel nacional, nosotros invisibilizábamos también a los grupos indígenas al interior de la Universidad." (Entrevista. En Sierra, 2004: 259).

Ante esta ausencia de propuestas educativas pertinentes a sus contextos, distintas organizaciones étnicas de Colombia y Latinoamérica vienen impulsando sus propios proyectos de educación post-secundaria hacia el fortalecimiento de los procesos organizativos comunitarios. Resaltamos aquí la Universidad Autónoma Indígena Intercultural (UAII) del Consejo Regional Indígena del Cauca (CRIC) de Colombia, cuyo proceso educativo está orientado a:

El trabajo en la formación de líderes, de dirigentes, así como de profesores, promotores de salud, multiplicadores organizativos, agentes de producción, comunicadores, múltiples personas encargadas de dinamizar, defender y apoyar los procesos comunitarios. Estas dinámicas formativas se han caracterizado por retomar los espacios escolarizados y los no escolarizados, por partir de las expectativas y potencialidades comunitarias, por desarrollar métodos, técnicas y en general un modelo que conjuga la sabiduría de nuestros pueblos con los conocimientos de otras culturas ${ }^{1}$.

Otros ejemplos son la Universidad de la Tierra en Chiapas-México (Moreno, 2007) y la Universidad Intercultural de las Nacionalidades y Pueblos Indígenas "Amawtay Wasi"en Ecuador (Sarango, 2009).

También encontramos a nivel internacional distintas experiencias significativas de alianzas entre universidades y organizaciones comunitarias

\footnotetext{
${ }^{1}$ Universidad Autónoma Indígena Intercultural. Disponible en: http://www.cric-colombia.org/portal/universidad-autonoma-indigena-intercultural-uaii/
} 
hacia la construcción conjunta de conocimiento o "desarrollo endógeno" (Haverkort \& Rist, 2007. Ver también CAPTURED ${ }^{2}$, AGRUCO $^{3}$, COMPAS $^{4}$ ). En Colombia, la autora, a través del Grupo de Investigación DIVERSER ${ }^{5}$ y la Facultad de Educación, ha impulsado en la Universidad de Antioquia distintos proyectos investigativos y educativos con comunidades afro, indígena y campesina, hacia la creación conjunta de programas en los mismos contextos comunitarios, entre ellos, la "Licenciatura en Pedagogía de la Madre Tierra", en convenio con la Organización Indígena de Antioquia ${ }^{6}$.

Estas y otras iniciativas nos abren horizontes para quienes queremos contribuir desde los ámbitos universitarios a construir de manera colaborativa y solidaria, proyectos investigativos y prácticas educativas hacia una sociedad más democrática, justa y equitativa.

\section{HACIA LA CREACIÓN COLABORATIVA ENTRE UNIVERSIDADES Y COMUNIDADES A TRAVÉS DEL PROYECTO “RURALIDAD DESDE LA EQUIDAD Y LA DIVERSIDAD"}

La tensión entre universidades convencionales y comunidades en contextos rurales continuará mientras no se den transformaciones profundas a la relación asimétrica históricamente establecida, en la cual las primeras son las poseedoras del saber mientras las segundas son ignoradas o convertidas en receptoras pasivas de prácticas docentes e investigativas, sin un reconocimiento real como interlocutoras válidas en los procesos de construcción y aplicación del conocimiento, de su papel activo en la transformación de las condiciones sociales, políticas y económicas que las asfixia y oprime.

La situación de la población rural en Colombia es crítica hoy día pues es quien ha sufrido principalmente los estragos del conflicto armado, la expropiación

\footnotetext{
${ }^{2}$ CAPTURED, Programa internacional para la construcción de capacidades y teorías en universidades y centros de investigación para el desarrollo endógeno sostenible), http://www.captured-la.org/index. php?option=com_content\&view=article\&id=67\&ltemid=62

${ }^{3}$ AGRUCO, Centro universitario de la Facultad de Ciencias Agrícolas, Pecuarias, Forestales y Veterinarias de la Universidad Mayor de San Simón, Bolivia, http://www.agruco.org/agruco/quiacnes-somos

${ }^{4}$ COMPAS, Comparing and Supporting Endogenous Development, http://www.compasnet.org/?page_id=22

${ }^{5}$ DIVERSER, Grupo de Investigación en Pedagogía y Diversidad Cultural de la Universidad de Antioquia. Ver ejemplos de algunas experiencias en Sierra y Romero; 2002; Sierra, 2005; Sierra, Siniguí y Henao, 2010; Sierra y Sierra, 2011; Colectivo pedagógico, 2012.
}

${ }^{6}$ Ver: www.pedagogiamadretierra.org 
territorial, el abandono estatal y la frecuente violación de sus derechos humanos fundamentales (UN Permanent Forum on Indigenous Issues, 2011; PNUD, 2011; Cruces, Casparini \& Carvajal, 2010, Mondragón, 2006; SEMANA, 2013). A pesar de su tamaño relativamente pequeño (menos del $1 \%$ de la superficie del planeta), Colombia es considerado uno de los diez países 'megadiversos' (Maffi, 1998; 2005), no solo por sus variados ecosistemas sino también por su diversidad cultural y lingüística. Cerca del 32\% de la población colombiana todavía vive en "áreas predominantemente rurales", y aproximadamente de 9 a 11 millones de sus habitantes son agricultores; entre ellos, las poblaciones indígenas y afrodescendientes, quienes representan el $2 \%$ y el $21 \%$ de la población total del país (estimada en 44 millones) respectivamente.

Es en los territorios indígenas, afro y mestizo campesino colombianos donde esta diversidad biocultural todavía se mantiene y que, después de décadas de resistencia, logró que en la reforma constitucional de 1991 se reconociera el carácter pluricultural de la nación y derechos específicos de los grupos étnicos y de pequeños agricultores en temas como autonomía territorial, fortaleza lingüística, programas educativos culturalmente pertinentes y auto-gobierno ${ }^{7}$. Sin embargo, una estructura colonial de despojo y tenencia de tierras, aunado a la creciente presión de intereses transnacionales para explotar materia prima y monocultivos, tienen hoy en alto riesgo la pervivencia de estas diversas culturas y sus ecosistemas, situación que no es exclusiva del contexto colombiano.

¿Qué papel -y desde qué paradigmas- se debe pensar una educación superior que responda a la situación de crisis que atraviesan comunidades rurales locales? Los autores de este artículo, en diálogo desde el año 2010, decidimos aunar esfuerzos desde nuestras universidades en Colombia y Canadá para hacer posible un proyecto de colaboración internacional que nos permita acceder a recursos y apoyar organizaciones de poblaciones étnicas y rurales que han manifestado su interés en la creación de programas de educación superior pertinentes a sus planes de vida. Nos interesa contribuir a la transformación de nuestras universidades desde paradigmas decoloniales; lo cual significa propiciar el trabajo conjunto entre colectivos "académicos" y "no-académicos", esto es, entre representantes de instituciones de educación formal y representantes de comunidades y organizaciones comunitarias (u otros actores sociales), responsables a su vez de complejos procesos educativos, aunque éstos no otorguen una certificación oficial.

Desde finales del año 2012, a través de distintos encuentros y entrevistas,

\footnotetext{
${ }^{7}$ Constitución Política de Colombia, Artículos 7, 10, 63, 68, 72, 246, 286.
} 
venimos conformando colectivos de trabajo transdisciplinarios en nuestras respectivas universidades hacia la conformación de una alianza con organizaciones étnico-territoriales del noroccidente colombiano (COCOMACIA ${ }^{8}$, ASOREWA ${ }^{9}$, CABILDO MAYOR INDÍGENA DE CHIGORODÓ ${ }^{10}$ ) y Organizaciones No Gubernamentales $\left(\mathrm{CEAM}^{11}, \mathrm{WWF}^{12}, \mathrm{OXFAM}^{13}\right)$, que previamente habían participado en proyectos anteriores, para el diseño e implementación de un proyecto investigativo y educativo que hemos denominado inicialmente "Hacia una ruralidad sostenible desde la equidad y la diversidad"14. También participan en el diálogo otras instituciones de educación superior de la región (FUCLA y UTCH). ${ }^{15}$

Esta propuesta de investigación busca profundizar en prácticas de participación comunitaria y construcción conjunta del conocimiento, dentro de un marco de colaboración transdisciplinaria, a través de la revitalización de los sistemas de conocimiento local sobre sostenibilidad. Por participación comunitaria nos referimos a un proceso de aprendizaje social a través del cual comunidades académicas y no académicas construyen relaciones progresivas equitativas para definir retos de sostenibilidad. Para ello retomaremos elementos de la Investigación Acción Participativa (IAP), proceso de construcción de conocimiento democrático hacia la conjunción de acción y reflexión, de teoría y práctica, con la participación de otros, en la búsqueda de soluciones prácticas a problemas que afectan a la gente y hacia el florecimiento de las personas y sus comunidades (Alcocer, 1998; Fals Borda, 1985, 1986a, 1986b; Selener, 1997; Reason \& Bradbury, 2006). La transdisciplinariedad trasciende la multi- y la inter- disciplinariedad entendidas como la suma de disciplinas, cada una con sus teorías, metodologías y métodos específicos; ella permite la inclusión de otras formas de conocimiento y de otras culturas a un nivel muy

\footnotetext{
${ }^{8}$ COCOMACIA, http://www.cocomacia.org.co/

${ }^{9}$ ASOREWA, http://www.orewa.org/index.php/sobre-nosotros

${ }^{10}$ Cabildo Mayor Indígena de Chigorodó, http://emberachigorodo.org/

${ }^{11}$ CEAM, Corporación de Estudios, Educación e Investigación Ambiental, http://www.corpoceam.org/

${ }^{12}$ WWF-Colombia, http://www.wwf.org.co/

${ }^{13}$ OXFAM-Colombia, http://www.oxfam.org/es/colombia

${ }^{14}$ Aspiramos presentar esta propuesta a convocatoria de colaboración internacional hacia la sostenibilidad del Consejo para la Investigación en Ciencias Sociales y Humanidades de Canadá (SSHRC) y otras entidades internacionales y nacionales.

${ }^{15}$ FUCLA: Fundación Universitaria Claretiana; UTCH:Universidad Tecnológica del Chocó “Diego Luis Córdoba"
} 
profundo, en lo que se conoce como el diálogo intercultural e inter-científico (Delgado \& Rist, 2011).

Desde este diálogo de saberes, proponemos examinar la formación en las actuales políticas y los programas oficiales de educación, con las siguientes preguntas: 1) ¿hasta dónde están o no contribuyendo con los procesos organizativos y comunitarios de poblaciones rurales y la sostenibilidad de sus ecosistemas? 2) ¿Cómo desde este proyecto podemos incidir en la transformación de políticas económicas, sociales y educativas que afectan el Buen Vivir de las comunidades rurales?

Otras preguntas a explorar de manera conjunta son: 3) ¿Qué consideran las comunidades participantes afecta su sostenibilidad, teniendo en cuenta su diversidad histórica, étnica, biogeográfica y cultural? ¿Bajo qué paradigmas socioculturales y de sostenibilidad deberían ser analizados e interpretados los principales problemas y retos de las comunidades participantes? 4) ¿Qué niveles de articulación existen entre las escuelas rurales y el currículo institucional en relación con los problemas y retos de sostenibilidad identificados con las comunidades? ¿Cuáles alternativas educativas sugieren las diversas comunidades, según su diversidad histórica, étnica, biogeográfica y cultural? 5) ¿Qué tipo de contenidos relacionados con el conocimiento local, se debe fortalecer o transformar; cuáles experticias académicas interdisciplinarias y tecnológicas deben ser adoptadas o transformadas, teniendo en cuenta los problemas actuales y los retos que diversas comunidades rurales enfrentan hoy?

Este proyecto incluirá la realización de una revisión comprensiva de los sistemas formales y no formales de la educación en Colombia, con representantes de comunidades afro, indígenas y mestizas campesinas, en relación a políticas y prácticas sobre sostenibilidad. El análisis participativo de políticas procura la vinculación en los procesos investigativos de aquellos afectados por las decisiones de quienes definen las políticas. Al Análisis Crítico Participativo de Políticas (ACPP) también se le conoce en la literatura como "análisis y definición participativos de políticas" (Geurts \& Joldersma, 2001) y se refiere a la participación de la comunidad en el análisis y definición de políticas (Petersen et al., 2006). Apoyados en teorías del discurso que permitan un análisis crítico de las políticas (Jørgensen \& Phillips, 2002), examinaremos documentos nacionales y departamentales de las políticas con sus respectivos programas educativos, teniendo en cuenta el origen e historia de las políticas; contextos sociales, políticos e institucionales; visiones orientadoras; 
fundamentos paradigmáticos; y estrategias para su implementación (Fallon \& Paquette, 2008).

El proceso participativo de análisis de políticas se orientará al fortalecimiento de las dinámicas de los sistemas de conocimiento endógeno. No pretendemos que la investigación participativa comunitaria sirva para estudiar el conocimiento endógeno desde una perspectiva de la sociedad dominante o para construir conocimiento externo sobre conocimiento endógeno, sino que este proyecto contribuya a revitalizar y fortalecer la base de conocimiento endógeno de las comunidades participantes hacia la sostenibilidad de sus planes de vida y el diálogo de saberes con la comunidad universitaria.

\section{DESAFÍOS HACIA LA CONSTRUCCIÓN CONJUNTA DE SABERES Y PROGRAMAS ENTRE COMUNIDADES Y UNIVERSIDADES}

El esfuerzo de crear alianzas de cooperación entre universidades y comunidades desde paradigmas decoloniales, no está libre de dificultades y desafíos pues significa romper una tradición histórica colonial de negación, deslegitimación y apropiación de saberes de las primeras hacia las segundas. A su vez, el aislamiento de las universidades otras formas de pensamiento y maneras colectivas de relacionarse contribuye a hacer más efectivos paradigmas neoliberales e individualistas en sus programas académicos. Universidades y comunidades requieren avanzar en un diálogo continuo recíproco hacia la construcción de sociedades más justas y equitativas. Desde nuestras experiencias, aunadas a las reflexiones de otras latitudes, brevemente mencionamos aquí algunas de las situaciones a estudiar y analizar con participantes en procesos conjuntos de formación en liderazgo comunitario:

Co-optación. Un temor frecuente de organizaciones y comunidades es la apropiación de sus iniciativas por parte de académicos u entidades estatales sin que haya una real transformación de las condiciones que les afectan; es el caso de incluir en la educación formal conceptos y propuestas de educación propia de las comunidades (bien sea denominándolas etnoeducación, educación intercultural, acción afirmativa o inclusiva), sin cambios sustanciales en el sistema educativo y la sociedad dominantes (Rebolledo, 2010; Wash 2009). En este sentido, Walsh (2010) se pregunta si un concepto como el "Buen Vivir" al ser adoptado e hibridizado por el Estado ecuatoriano, y al enmarcarlo en conceptos como "Desarrollo Humano Sostenible", pierde su fuerza radical al ser co-optado como una herramienta discursiva más, pero con poco significado 
para una real transformación intercultural, interepistémica y plurinacional. Asuntos sobre sostenibilidad, consulta previa, mejoramiento de la calidad de vida, saberes ancestrales terminan siendo cooptados por intereses ajenos a las mismas comunidades. ¿Cómo identificar tan dispares intereses? Ver preocupaciones similares de Gudynas (2013) sobre las contradicciones del gobierno de Bolivia respecto a las políticas del "Buen Vivir", al justificar explorar y explotar hidrocarburos en zonas que permanecían resguardadas por ser tierras de comunidades campesinas e indígenas o áreas naturales protegidas.

Esencializar, satanizar o romantizar al "Otro". La comunicación se rompe cuando se acepta o ignora el concepto de una persona sólo por su origen étnico y social, escolaridad, género o edad, sin dar oportunidad a escuchar y profundizar en los argumentos que se expresan. En ocasiones se "satanizan" voces de personas de la academia, vengan del Norte o el Sur, así hagan propuestas pertinentes o se asume como autoridad moral, sin criticismo, todo planteamiento que provenga de representantes de los grupos étnicos o poblaciones más oprimidas, sin reconocer que hay diferencias entre líderes y miembros de un mismo grupo poblacional y que la herencia colonial nos ha afectado por igual a todas y todos. ¿Cómo sopesar la validez de un argumento o una postura ética, política, educativa o filosófica? En palabras del colectivo de CAPTURED,

Se debe procurar que estudiantes e investigadores no glorifiquen, romanticen o rechacen los conocimientos endógenos o los convencionales... Encontrar la justa distancia y una actitud constructiva y crítica, tanto hacia el conocimiento endógeno como el convencional, es un proceso de aprendizaje importante. (Haverkort et al, 2012, p. 20)

La auto-reflexividad. Enfrentar esencialismos y estigmatismos exige no perder de vista el horizonte al que se quiere llegar; el esfuerzo por derrumbar conjuntamente barreras de discriminación y construir en alianza el sueño de una sociedad más equitativa, solidaria y pluricultural. Para ello es necesario mantener la apertura al diálogo como un acto pedagógico en sí mismo, y continuamente aplicar en nosotros -individual y colectivamente- la autoreflexividad. Para develar el pensamiento etnocentrista, Matsumoto (2000) nos invita a explorar en la propia cultura las razones históricas y sociales de los prejuicios y la discriminación. Una mayor sensibilidad intercultural significa un permanente proceso de descentración, esto es, reconocer los límites de nuestro propio conocimiento; para Varela (1981) ello es posible porque 
"nuestras descripciones son capaces de autodescripción" (p. 258-261), esto es, somos capaces de hacer descripciones de nosotros mismos, de nuestro propio conocer. Conocemos entonces por la posibilidad de la participación y la interpretación; pasamos así del plano de la esencialidad al plano de la autorreflexividad, esto es, "poner de relieve las estereotipadas propiedades de la realidad que se engendran cuando uno cree estar en posesión de la verdadera y definitiva explicación del mundo" (Watzlawick, 1981:141). ¿Cómo saber qué debemos retener o cambiar de los valores y concepciones de la cultura en la cual hemos crecido?

Condiciones simétricas y asimétricas para el diálogo de saberes. Es importante reconocer las disímiles condiciones de las personas o grupos que entran a participar de un colectivo o una alianza. En condiciones simétricas, en las cuales las personas se reconocen como pares equivalentes e interlocutores igualmente válidos, considerar distintos puntos de vista se convierte en un excelente ejercicio cognitivo y dialógico, de intercambio de ideas, de aprendizaje mutuo. La inteligencia y la creatividad afloran y es posible la cooperación y el esfuerzo conjunto para emprender acciones, sin que ello niegue la posibilidad del disenso y la crítica. Cuando la relación es asimétrica y no se generan condiciones para la reflexión sobre el origen y devenir de las diferencias y, por ende, de las relaciones de poder en juego, predomina la imposición de ideas y el autoritarismo por una parte, y la resistencia y la frustración por otra, ante el no logro de objetivos. Esto puede aplicarse tanto al encuentro entre naciones, pueblos o grupos étnicos (dominantes y alternos) como a relaciones de corte más micro, como las que se establecen en los ámbitos familiar, escolar o comunitario.

Mientras se resuelven condiciones asimétricas, ¿cómo generar la complicidady solidaridaden la búsqueda de objetivos comunes? ¿Son posibles el diálogo y la reflexión en situaciones asimétricas como las que actualmente existen entre universidades y comunidades? Pensamos que sí pueden ser posibles, sólo si se acepta una continua auto-reflexividad, una mirada crítica e histórica, especialmente de quienes han ostentado una situación de privilegio, que permita indagar por el origen de dicha relación de no-equivalencia; significa atreverse a pensar que el mundo en el que hemos crecido y nos enseñaron a asumir como natural es un mundo socialmente construido $\mathrm{y}$, como tal, puede y debe cambiar, para desde allí generar objetivos comunes, procesos solidarios conjuntos. Para Palermo (2010), la búsqueda de la liberación de los sectores subsumidos y la equidad de todos los seres humanos, se requiere, 
...la "analéctica", lo que en la línea de las geopolíticas del conocimiento se ha definido como un a través de fronteras en busca de la "mutua fertilización creativa". Este tipo de relación dialógica, no obstante, no borra las contradicciones, las diferencias, las luchas, pues son ellas precisamente las que hacen posible la emergencia de la otredad y su reconocimiento (Palermo, 2010:53)

Naturalización de la institucionalidad. La universidad que conocemos no se rige por normas "naturales" sino socialmente construidas, algo que parece obvio pero que cotidianamente se olvida pues, ante una acción que se propone fuera de los marcos establecido, los funcionarios responden: "no se puede porque la norma no lo permite". Se olvida que son personas quienes crean las normas y son personas quienes las pueden cambiar. Un ejemplo fue el largo proceso de gestión para el cambio de normatividad para el reconocimiento de las lenguas nativas en la Universidad de Antioquia (2002-2008), y por ende, del castellano -no el inglés- como segunda lengua para la admisión de estudiantes indígenas y raizales a programas de posgrado. Para generar procesos de cambio en las universidades debe acompañarnos una constante reflexión socio-histórica y cultural que nos recuerde el papel que han jugado las instituciones oficiales en la reproducción de la opresión y la exclusión.

Sensibilidad hacia otras maneras de pensar el mundo. Con Haverkort et al (2012) compartimos que la mayoría del personal universitario solo ha recibido formación desde perspectivas dominantes de la ciencia anglo-europea y muy pocos están convencidos del valor del desarrollo endógeno; falta además comprensión de conceptos, fortalezas y debilidades del conocimiento endógeno así como de las actitudes y habilidades necesarias para enseñar desde esta perspectiva. Para que una universidad pueda hacer la transición de ser un agente de la ciencia dominante a una universidad que combina la ciencia anglo-europea con la educación y la investigación endógena de los pueblos, deben desarrollarse capacidades y sensibilidades de profesores y estudiantes en este sentido; en particular, de jóvenes profesionales para que puedan apoyar la educación y la investigación endógena. Para estos autores,

Definirse por el desarrollo endógeno y la creación conjunta (co-creación) de distintas formas de conocimiento implica un número de cambios de la manera como la ciencia se viene desarrollando en el mundo; implica un cambio desde la nación de una ciencia superior y universal que reemplaza a otras ciencias hacia la noción de una pluralidad de ciencias, cada una con sus fortalezas y debilidades. Un cambio que vaya de una sola forma de adoptar la ciencia dominante, hacia la búsqueda del mejoramiento de 
diferentes ciencias a través del aprendizaje y acción al interior de cada ciencia, y el intercambio y aprendizaje conjunto entre-ciencias. Es un cambio de la lógica de la exclusión a la lógica de la inclusión, de la visión de una sola disciplina científica a la de la trans-disciplinariedad y la investigación acción participativa. (p. 28. Traducción nuestra).

\section{A MODO DE CONCLUSIÓN}

Abordamos en este artículo distintos paradigmas sobre la sociedad y la cultura, y cómo distintas maneras de concebir el mundo impactan la formación de futuros profesionales. La universidad imperante hoy día se rige por paradigmas individualistas, competitivos y de la ganancia personal, en contravía de paradigmas decoloniales y simbiosinérgicos, basados en la crítica y transformación de los procesos de opresión que afectan a comunidades enteras, hacia la búsqueda del bien común y el cuidado de la naturaleza. Tanto la educación básica como universitaria necesitan ser transformadas, de tal forma que la indagación trans-disciplinaria, esencial para la comprensión de entornos socio-ecológicos, culturales, políticos y económicos complejos, lleguen a ser la norma y no la excepción; de esta manera, que el sector educativo esté mucho más integrado a otros sectores sociales que vienen afrontando los impactos de una relación humana depredadora con el medio ambiente.

Es necesario transformar entonces el carácter monocultural de la universidad para que podamos aprender de otras maneras de ser, sentir, pensar y habitar el mundo, lo cual no está exento de problemas y desafíos. En palabras de CastroGómez (2005),

La pluralidad epistémica [...] no es posible ni pensable sin una democracia epistémica en la que la ciencia deje de ser una sierva del capital y en la que diversas formas de producir y transmitir conocimientos puedan coexistir y complementarse. Me refiero a un mundo en el que sistemas no occidentales de conocimiento puedan ser incorporados en los currículos de universidades occidentales, y en igualdad de condiciones, en ámbitos tales como el derecho, la medicina, la biología, la economía y la filosofía. Un mundo en el que, por ejemplo, la cosmovisión Yoruba, la cosmovisión del budismo Zen, o la cosmovisión de los indios cunas, pueda servir para avanzar hacia una ciencia más integral, más orgánica, más centrada en lo común y no en las necesidades del capital. Quizás, entonces, sólo entonces, podamos avanzar hacia un mundo en que muchos otros mundos sean posibles. 


\section{RECONOCIMIENTOS}

A nuestras instituciones (Universidad de Antioquia y University of British Columbia) por el respaldo a esta iniciativa de cooperación conjunta. Avances de estas reflexiones se presentaron en el IX Congreso Internacional de Investigación Cualitativa, realizado en la Universidad de Illinois, Urbana, Estados Unidos, Mayo 15 al 18 de 2013; agradecemos las apreciaciones allí recibidas. Nuestros reconocimientos también a las Dras. Alma Rivera (Universidad Iberoamericana de México) y Gricelda Figueroa (Universidad Santiago de Chile) por sus observaciones y sugerencias a versiones previas. El escrito final es de nuestra entera responsabilidad.

\section{REFERENCIAS}

ALCOCER, Marta, "Investigación acción participativa", En Galindo Cáceres, Luis Jesús (Ed.), Técnicas de investigación en sociedad, cultura y comunicación (pp. 433-464), Prentice Hall, México, 1998.

BALL. Stephen, Global Education Inc.: New policy networks and the neoliberal imaginary, Routledge, London, 2012.

BERTRAND, Yves, VALOIS, Pierre, Les options en éducation. Ministère

de l'Éducation, Québec, 1980.

CASTRO-GÓMEZ, Santiago, Ciencias sociales, violencia epistémica y el problema de la "invención del otro", En Lander, Edgardo (Ed.), La colonialidad del saber: eurocentrismo y ciencias sociales. Perspectivas latinoamericanas (pp. 145-163), CLACSO-UNESCO Buenos Aires, 2000, Disponible en: http:// bibliotecavirtual.clacso.org.ar/ar/libros/lander/lander.html.

CASTRO-GÓMEZ, Santiago, El capítulo faltante de Imperio. La reorganización posmoderna dela colonialidad en elcapitalismo posfordista, Artículo presentado en el Simposio Internacional “¿Uno solo o varios mundos posibles?” organizado por el Instituto de Estudios Sociales Contemporáneos (IESCO), Bogotá, junio 7-10 de 2005. Disponible en Multitudes. http://multitudes.samizdat.net/Elcapitulo-faltante-de-Imperio-La.

CAUDILLO, Gloria Alicia, "El buen vivir: un diálogo intercultural", Ra-Ximahi, Vol. 8, no 2, 2012, ps. 345-364.

CHOQUEHUANCA, David, "25 postulados para entender el "Vivir Bien" - 
Entrevista a David Choquehuanca", La Razón de Bolivia, 2010, Disponible en: http://indigenaslibertarios.blogcindario.com/2010/02/00055-25-postuladospara entender el-vivir-bien-entrevista-con-david-choquehuanca-bolivia.html.

COLECTIVO INTERCULTURAL, “¿Por qué la realización de este estudio?

Insatisfacción con la formación universitaria para los pueblos indígenas", Revista Educación y Pedagogía, Facultad de Educación Universidad de Antioquia, Vol. XIX, No. 49, 2007, ps. 21-39.

COLECTIVO PEDAGÓGICO, "Diploma en Gestión del Territorio para pueblos indígenas y afrodescendientes", Informe Final, Grupo de Investigación Diverser de la Universidad de Antioquia, WWF, Oxfam y Organizaciones ÉtnicoTerritoriales del Pacífico Colombiano, 2012.

CORONIL, Fernando, Naturaleza del poscolonialismo: del eurocentrismo al globocentrismo, En Lander, Edgardo (Ed.), La colonialidad del saber: eurocentrismo y ciencias sociales. Perspectivas latinoamericanas (pp. 87-107), CLACSO-UNESCO Buenos Aires, 2000.

DELGADO, Fernando y RIST, Stephan, La transdisciplinariedad y la investigación participativa en una perspectiva de diálogo intercultural $e$ intercientifico, Working document, AGRUCO/CAPTURED, La Paz, Bolivia, 2011.

ESCOBAR, Arturo, La invención del Tercer Mundo: Construcción y deconstrucción del desarrollo, Norma, Bogotá, 1998.

ESCOBAR, Arturo, "Beyond the Third World: Imperial globality, global coloniality and antiglobalisation social movements", Third World Quarterly, Vol. 25, no 1, 2004, ps. 207-230.

ESCOBAR, Arturo, "Worlds and knowledges otherwise. The Latin American

modernity / coloniality research program", Cultural Studies, Vol. 21, no 2-3, 2007, ps. 179-210.

FALLON, Gerald. y PAQUETTE, Jerald, "Devolution, choice, and accountability in the provision of public education in British Columbia: A critical analysis of the School Amendment Act of 2002 (Bill 34)", Canadian Journal of Educational Administration and Policy, Vol. 75, 2008, ps. 1-16. [Online] http://www. umanitoba.ca/publications/cjeap/articles/fallonpaquette.html.

FALS BORDA, Orlando, Conocimiento y Poder Popular, Siglo XXI, Bogotá, 1985.

FALS BORDA, Orlando, Investigación participativa (con Carlos R. Brandao). Instituto del Hombre, Montevideo, 1986a.

FALS BORDA, Orlando, "La investigación-acción participativa: Política y epistemología”, en Camacho, Álvaro (ed.), (pp. 21-38), La Colombia de hoy, 
Cerec, Bogotá, 1986b,

GARCÍA CASTANO, Javier., PULIDO MOYANO, Rafael, y MONTES DEL CASTILLO, Ángel, La educación multicultural y el concepto de cultura, En García Castaño, Javier \& A. Granados Martínez, Antolín (Eds.), Lecturas para educación intercultura/(pp. 47-80), Trotta, Madrid, 1999.

GEURTS, Jac L.A., y JOLDERSMA, Cisca, "Methodology for participatory policy analysis". European Journal of Operational Research, Vol. 128, 2001, ps. 300-310

GLAVEANU, Vlad Petre, "Paradigms in the study of creativity: Introducing the perspective of cultural psychology", New Ideas in Psychology, Vol. XXX, 2009, ps. 1-15.

GUDYNAS, Eduardo, Buen Vivir: germinando alternativas de desarrollo, Agencia Latinoamericana de Información (ALAI,), Quito, 2011. Disponible en: http://alainet.org/contacto.phtml

GUDYNAS, Eduardo, "A explotar, a compensar, a concientizar", Blog Embrollo del desarrollo. Bogotá: Periódico El Espectador, 2013. Disponible en: http:// blogs.elespectador.com/embrollodeldesarrollo/

HARDING, Sandra., Ciencia y feminismo. Morata, Madrid, 1996.

HARDT, Michael y NEGRI, Toni, Imperio, Ediciones desde abajo, Bogotá, 2001.

HAVERKORT, Bertus, y RIST, Stephan (Eds.), Endogenous development and bio cultural diversity: The interplay of worldviews, globalization and localization. COMPAS, Leusden, 2007.

HAVERKORT, Bertus, MILLAR, David, SHANKAR, Darshan, y DELGADO, Freddy, Intra- and Inter-science dialogues : towards co-creation of sciences Different sciences and their possible levels of co-creation. (Chapter 6-Conclusion) Towards the co-construction of sciences. (copia en Word gentilmente cedida por los autores), 2012.

JØRGENSEN, Marianne y PHILLIPS, Louise, Discourse Analysis as Theory and Method. Sage Publications, London, 2002.

LAMUS, D., "Race and ethnicity, sex and gender: The meaning of difference and power", Reflexión Política (Universidad Autónoma de Bucaramanga, Colombia), Vol. 14, no 27, 2012, ps. 69-84.

LANDER, Edgardo, Ciencias sociales: saberes coloniales y eurocéntricos, En Lander, Edgardo (Ed.), La colonialidad del saber: eurocentrismo y ciencias sociales. Perspectivas latinoamericanas (pp. 11-41), CLACSO-UNESCO, Buenos Aires, 2000. Disponible en: http://bibliotecavirtual.clacso.org.ar/ar/libros/ 
lander/lander.html

LEON, Magdalena y Holguín, Jimena, Acción Afirmativa: Hacia democracias inclusivas (COLOMBIA). Fundación Equitas, Santiago, Chile, 2005. Disponible en: http://issuu.com/fundacion.equitas/docs/aa_colombia.

MAFFI, Luisa, "Language: A Resource for Nature", The UNESCO Journal on the Environment and National Resources Research, Vol. 34, no 4, 1998, ps.1221.

MAFFI, Luisa, "Linguistic, Cultural, and Biological Diversity", Annual Review of Anthropology, Vol. 29, 2005, ps. 599-617.

MONDRAGON, Héctor, Colombia: Agrarian Reform-Fake and Genuine. En Peter Rosset, Raj Patel, Michael Courville (Eds.). Promised land: competing visions of agrarian reform [164-176]. Institute for Food and Development Policies, New York, 2006. Available in: http://www.foodfirst.org/files/ bookstore/pdf/promisedland/8.pdf

MORENO, Alejandro, La Universidad de la Tierra en Chiapas, 2007. Disponible en: http://alejandromorenolax.blogspot.com/2007_12_01_archive.html.

NIETO, Mauricio, Remedios para el imperio: Historia natural y la apropiación del nuevo mundo, Instituto Colombiano de Antropología e Historia (ICANH), Bogotá, 2000. Disponible en: http://historiadelaciencia-mnieto.uniandes.edu. co/html/lib.html.

PALERMO, Zulma, "La universidad latinoamericana en la encrucijada decolonial", Otros Logos, Revista de Estudios Críticos, Universidad Nacional del Comahue, Argentina., Año 1, No. 1, 2010, ps. 43-69.

PANCHO, Avelina, BOLANOS, Graciela., MANIOS, Socorro., CHAVACO, Antonio José., VILUCHE, Joaquin., SISCO, Manuel, et al, Educación Superior Indigena en Colombia. Una apuesta de futuro y esperanza. IEASALC-UNESCO, ASCUN, CRIC, MEN, ONIC, Cali, 2005. Disponible en http://www.humanas.unal. edu.co/colantropos/documentos/EducSupIndigena.pdf

PAQUETTE, Jerald y FALLON, Gerald, (2010), First Nations Education Policy in Canada. University of Toronto Press, Toronto, 2010.

PETERSEN, Dana., MINKLER, Meredith, Breckwich Vásquez, Victoria, and BADEN, Andrea Corage, "Community-based participatory research as a tool for policy change: A case study of the Southern California Environmental Justice Collaborative". Review of Policy Research, Vol. 23, no 2, 2006, ps. 339-53.

PLOEG, Jan Douwe van der, Nuevos campesinos: Campesinos e imperios alimentarios, Icaria Barcelona, 2010.

PNUD, 2011. Colombia rural: razones para la esperanza. Informe 
Nacional de Desarrollo Humano 2011. Machado, Absalón (Ed.). Programa de las Naciones Unidas para el Desarrollo (PNUD), Bogotá, 2011. Disponible en: $\quad$ http://www.undp.org/content/dam/undp/documents/projects/ COL/00056950/2Anexo\%20sobre\%20proceso\%20de\%20difusi\%C3\%B3n\%20 INDH\%20-\%20Informe\%20a\%20Embajada\%20de\%20Suecia\%20(2).pdf

REASON, Peter y BRADBURY, Hilary, Inquiry \& participation in search of a world worthy of human aspiration, 2006. Retrieved January 23, 2013, from: http://faculty.mu.edu.sa/public/uploads/1346012794.621handbook_of_ action_research.pdf

REBOLLEDO, Nicanor, Interculturalismo y autonomía. Las universidades indígenas y las políticas de alteridad. En Navarro Gallegos, César (coord.), La mala educación en tiempos de la derecha. Porrúa, México, 2005.

RESTREPO, Darío, De la falacia neoliberal a la nueva política, En D. Restrepo Botero (Ed.), La falacia neoliberal: crítica y alternativas (pp. 17-40). Universidad Nacional de Colombia, Bogotá, 2003.

SARANGO, Luis Fernando, La experiencia de la Universidad Intercultural de las Nacionalidades y Pueblos Indigenas "Amawtay Wasi": Hacia un Nuevo Paradigma de Educación Superior, Conferencia presentada en el XII Congreso ARIC. Florianópolis, Brasil, 2009. Disponible en: http://aric.edugraf.ufsc.br/ congrio/html/anais/anais.html ARIC 2009.

SELENER, Daniel, Participatory Action Research and Social Change: Cornell Participatory Action. Research Network, Cornell University, Ithaca, 1997.

SEMANA, Revista, "Cinco millones de víctimas y contando..." Proyecto Víctimas, Informe especial, Bogotá, 2013. Disponible en: http://www.semana. com/Especiales/proyectovictimas/index.html

SIERRA, Zayda, ¿Por qué el modelo educativo aculturizante de nuestras universidades? En Z. Sierra (Ed.), "Voces indigenas universitarias: expectativas, vivencias y sueños" (pp. 241-270), Grupo Diverser-Universidad de Antioquia, Colciencias, IESALC_UNESCO, OIA y otras entidades, Medellín, 2004.

SIERRA, Zayda, "Estudiantes indígenas en la universidad: ¿Qué modelo educativo caracteriza su formación?" Revista Colombiana de Educación, Vol. 48, 2005, ps. 177-195.

SIERRA, Zayda y ROMERO, Luz Angélica, "¿Investigar o construir nuevas realidades escolares? Reflexiones a propósito de un proceso formativo e investigativo con docentes en San Onofre, Sucre," Revista Colciencias. Colombia, Ciencia \& Tecnología, Vol. 20, no 4, 2002, ps. 19-32.

SIERRA, Zayda y SIERRA, Rodolfo, "Pedagogía del Agua y la Vida", proyecto 
pedagógico investigativo en contextos rurales del oriente antioqueño con estudiantes de Licenciatura en Educación Infantil, Facultad de Educación, Universidad de Antioquia, 2011.

SIERRA, Zayda; SINIGUI, Sabine y HENAO, Alexandra, "Acortando la distancia entre la escuela y la comunidad - Experiencia de construcción de un currículo intercultural en la Institución Educativa Karmata Rúa del Resguardo Indígena de Cristianía, Colombia", Visão Global (Universidad do Oeste de Santa Catarina), Vol. 13, no. 1, jan./jun 2010, ps. 219-252. Disponible en: http://editora.unoesc. edu.br/index.php/visaoglobal/article/viewFile/773/373

SMYTH, John, "Schooling and the enterprise culture: Pause for a critical policy analysis", Journal of Education Policy, Vol. 14, no 4, 1999, ps. 435-44.

UNESCO/IESALC, La educación superior indigena en América Latina, Instituto Internacional de la UNESCO para la Educación Superior en América Latina y el Caribe, Caracas, 2003.

WALSH, Catherine. (2009). Interculturalidad y (de)colonialidad: Perspectivas críticas y políticas. Artículo presentado en el XII Congreso ARIC. Brasil, Florianópolis, 2009. Disponible en: http://aric.edugraf.ufsc.br/congrio/html/ anais/anais.html

WALSH, Catherine, "Development as BuenVivir: Institutional arrangements and (de)colonial entanglements, Development, Vol. 53, no 1, 2010, ps.15-21.

\section{Zayda Sierra}

zsierra.udea@gmail.com

Exbecaria Fulbright y Doctora en Psicología Educativa, Universidad de Georgia, Estados Unidos. Profesora titular de la Facultad de Educación, Universidad de Antioquia, Medellín, Colombia. Fundadora y directora (19992011) del Grupo de Investigación Diverser (en Pedagogía y Diversidad Cultural) en esta misma universidad.

\section{Gerald Fallon}

gerald.fallon@ubc.ca

Doctor en Educación, University of Western Ontario, Canadá. Profesor Asistente, Departamento de Estudios Sociales de la Educación, University of British Columbia, Vancouver, Canadá. 